\title{
Scissor-type knife improves the safety of endoscopic submucosal dissection (ESD) among endoscopists without experience in ESD: a randomized ex vivo study
}

\section{다(1)우우}

\author{
Authors \\ Institutions \\ 1 Digestive and Liver Diseases, Columbia University \\ Medical Center-New York Presbyterian Hospital, New \\ York, New York, United States \\ 2 Division of Digestive and Liver Diseases, University of \\ Texas Southwestern, Dallas, Texas, United States \\ 3 Division of Gastroenterology and Hepatology, Mayo \\ Clinic, Rochester, Minnesota, United States \\ 4 Division of Gastroenterology, Washington University \\ School of Medicine, St. Louis, Missouri, United States
}

Kavel Visrodia', Tarek Sawas ${ }^{2}$, Liam Zakko³ ${ }^{3}$, Juan Reyes Genere ${ }^{4}$, Cadman Leggett ${ }^{3}$, Lori Lutzke ${ }^{3}$, Kenneth K. Wang ${ }^{3}$

\section{ABSTRACT} ESD. submitted 20.7.2020

accepted after revision 19.11 .2020

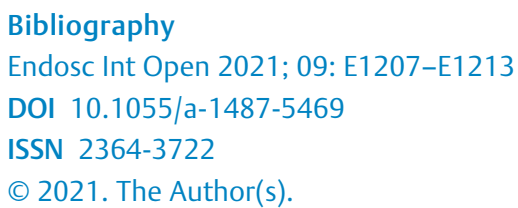

This is an open access article published by Thieme under the terms of the Creative Commons Attribution-NonDerivative-NonCommercial License, permitting copying and reproduction so long as the original work is given appropriate credit. Contents may not be used for commercial purposes, or adapted, remixed, transformed or built upon. (https://creativecommons.org/licenses/by-nc-nd/4.0/)

Georg Thieme Verlag KG, Rüdigerstraße 14,

70469 Stuttgart, Germany

Corresponding author

Kenneth K. Wang, MD, Division of Gastroenterology and Hepatology, Mayo Clinic, 200 First Ave. SW, Rochester, MN 55905, United States

Fax: +1-507-255-7612

wang.kenneth@mayo.edu
Background and study aims Endoscopic submucosal dissection (ESD) is technically challenging, difficult to learn, and carries a substantial risk of perforation, all of which remain significant barriers to its adoptability. We aimed to determine whether use of a novel scissor-type knife improved efficacy and safety among novice performers of

Materials and methods Following a brief didactic session on ESD, participants performed ESD of two lesions ( $2 \mathrm{~cm}$ diameter) in an ex vivo porcine gastric model. One resection was performed with a conventional knife and the other with the scissor knife (order of knife randomized). We recorded procedure time, successful en bloc resection, and adverse events (including full-thickness perforation and muscle injury) for each dissection. Participants completed a poststudy survey.

Results 10 endoscopists (8 trainees, 2 staff) considered novices in ESD participated. Compared with the conventional knife, use of the scissor knife was associated with a significantly shorter time to completion of submucosal dissection (mean 6.2 [SD 5.6] vs. 15.6 [SD 15.6] minutes; $P=$ 0.04 ) and total procedure time was not significantly different (22.1 [SD 13.3] vs. 24.9 [SD 26.5] minutes; $P=0.65$ ). Scissor knife use was also associated with a significantly lower proportion of perforation and/or muscle injury $(10.0 \%$ vs. $70.0 \% ; P<0.01)$ and proportion of muscle injury alone $(10.0 \%$ vs. $60.0 \%$; $P=0.02)$.

Conclusions Among novices performing ESD on an ex vivo animal model, use of a scissor knife was associated with a significantly lower proportion of adverse events without prolonging procedure time. Scissor-type knives may improve ESD safety, at least among novices.

\section{Introduction}

Endoscopic submucosal dissection (ESD) is a technique that allows for curative en bloc resection of large gastrointestinal (GI) lesions. ESD was pioneered in Japan over a decade ago for more optimal treatment of early gastric cancer given the inherent limitations of endoscopic mucosal resection (EMR). As ESD techniques have been refined and successfully applied throughout the GI tract, ESD has become integral to the endoscopic management of $\mathrm{Gl}$ pathology in Eastern countries.

ESD in the West, however, has not undergone a similar rate of adoption. ESD is technically challenging with a steep learning 
curve and carries a substantial risk of perforation, particularly in inexperienced hands, all of which remain significant barriers to its dissemination [1]. Dedicated ESD fellowships, which are widely available in Japan, have yet to be offered in the United States and would not be practical for endoscopists already in practice [2]. Therefore, to improve the adoptability of ESD, substantial efforts have been made to enhance the safety while facilitating the performance of ESD in the hands of trainees. This has resulted in the development of new knives and various other accessories.

One new category of ESD knife with a scissor-type tip configuration was introduced in Japan in the early 2010 s, and more recently these knives have been approved for use in the United States (- Fig. 1) [3-7]. Unlike conventional ESD knives, scissortype knives allow for tissue grasping prior to application of the electrosurgical current. This may help improve cutting precision and facilitate ESD, particularly in the hands of trainees. Although there is a growing body of literature supporting the safety and efficacy of these knives with ESD, this is primarily among supervised or experienced operators in Japan [3,8-10]. We aimed to determine whether use of one such scissor knife, the Clutch Cutter (Fujifilm Co., Tokyo, Japan), could improve the safety and efficacy among novice performers of ESD in an ex vivo model at a Western training institution.

\section{Materials and methods}

\section{Study design}

This was a prospective, randomized controlled study in which two $20 \times 20 \mathrm{~mm}$ lesions were created for ESD in an ex vivo porcine stomach model by participants without experience in ESD. Participants were blindly randomized in a 1:1 ratio using block randomization to use either the scissor knife or a conventional knife (HookKnife; Olympus Endotherapy, Tokyo, Japan) for the first ESD before crossing over and using the alternate knife for the second ESD ( $\triangleright$ Fig. 2 ). The study was approved by the Institutional Review Board (protocol \#17-000966) and all participants provided written consent.

\section{Participants}

Study participants were recruited by mass email to all current second- and third-year gastroenterology fellows and junior gastroenterology attending physicians. Participation was voluntary without remuneration. Requirements for study participation were: 1) no prior experience of performing ESD or using ESD knives, and 2) experience performing EMR (minimum 5) in the $\mathrm{Gl}$ tract. Prior to study participation, participants individually received a 30 minute, standardized didactic delivered by the study coordinator (K.V.) outlining relevant core ESD concepts including ESD theory, gastric wall anatomy, and tissue planes, basic ESD technique, and avoidance and recognition of adverse events. Videos demonstrating properties and appropriate technique of the two study knives were also reviewed. The didactic was followed by a hands-on opportunity to practice using each ESD knife in the animal model.

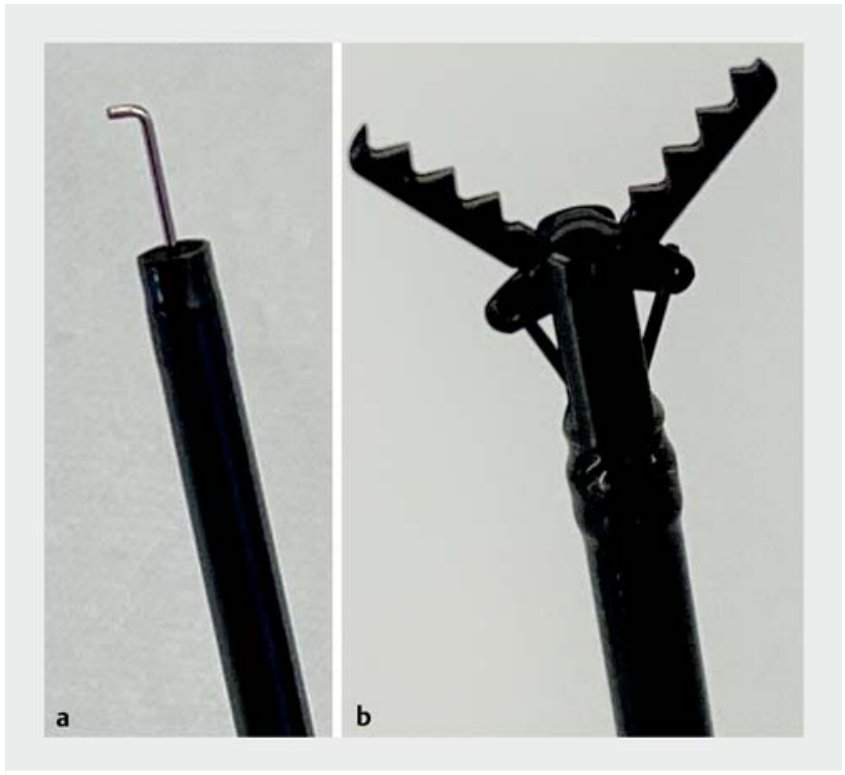

- Fig. 1 a Conventional knife (HookKnife; Olympus Endotherapy, Tokyo, Japan). b Scissor knife, 5.0 mm length (Clutch Cutter knife; Fujifilm Co., Tokyo, Japan).

Novice ESD performers (GI trainees/staff)

$\checkmark$

Instructional video and hands-on practice

ESD of two $2 \mathrm{~cm}$ lesions in an ex vivo porcine model

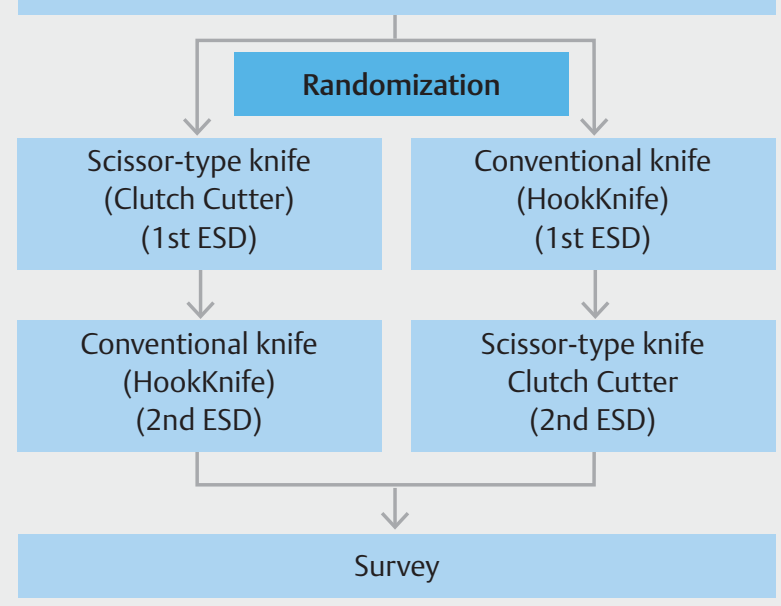

- Fig. 2 Study flow. ESD, endoscopic submucosal dissection; GI, gastrointestinal. Clutch Cutter knife (Fujifilm Co., Tokyo, Japan); HookKnife (Olympus Endotherapy, Tokyo, Japan).

\section{Ex vivo model}

All ESDs were performed in an ex vivo porcine stomach model ( $\triangleright$ Fig.3). Previously procured and frozen stomachs were defrosted overnight and prepared the morning of study procedures, including gastric lavage and setup in the animal model tray (Endo X Trainer; Medical Innovations International Inc., Ro- 


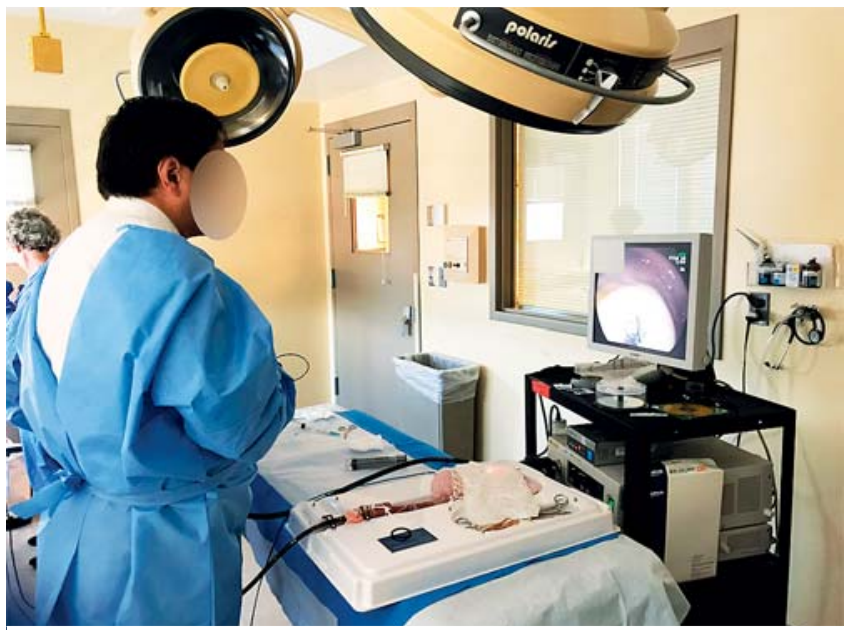

- Fig. 3 Setup of ex vivo porcine gastric model in animal laboratory.

chester, Minnesota, USA). Endoscopic equipment consisted of a gastroscope (Fujinon EG-450; Fujifilm) fitted with a soft transparent cap (Fujifilm) and an electrosurgical unit (VIO 300D; ERBE, Elektromedizin, GmbH, Tübingen, Germany).

The Clutch Cutter scissor knife is available in two lengths: $3.5 \mathrm{~mm}$ and $5.0 \mathrm{~mm}$. The $5.0 \mathrm{~mm}$ length was chosen for the study to facilitate mucosal incision and circumferential incision of relatively thick porcine gastric mucosa. Although several ESD knives with varying properties are available, the HookKnife was selected to represent conventional knives because of its longstanding use within the ESD community and properties similar to the Clutch Cutter including rotational and traction features, and its ability to create an initial mucosal incision prior to submucosal dissection (allowing for one knife to be used for the entire resection). Electrosurgical settings for both knives were determined based on performance in the animal model and as follows: Endo Cut Q mode, Effect 1, Cut duration 4, Cut interval 1. Functionality of this animal model was verified by an endoscopist with expertise in performing ESD (K.K.W.).

The study coordinator prepared both lesions for ESD by selecting a suitable area in the stomach, preferably along the greater curve or anteriorly in the body or antrum in an anti-dependent position, allowing gravity to aid in resection. Using a snare tip, border markings were made to create two neighboring lesions $20 \mathrm{~mm}$ in diameter. A coin $18 \mathrm{~mm}$ in diameter, which had been inserted during setup, was used for approximation of lesion size. A lesion size of $20 \mathrm{~mm}$ was chosen because this is the upper limit of a lesion that is resectable en bloc by EMR, and that a novice may consider for ESD.

\section{Procedure}

Participants prepared the lesion for ESD by submucosal injection of a premixed agent consisting of water, sodium chloride, medium-chain triglycerides, polyoxyl-15-hydroxystearate, poloxamer 188, and methylene blue (Eleview; Cosmo Pharmaceuticals NV, Dublin, Ireland) using an injection needle (Carr-Locke Injection Needle; US Endoscopy, Mentor, Ohio, USA) until adequate lift was achieved. Participants then proceeded with the first ESD using the designated knife. First, an initial mucosal incision was created to enter the submucosal plane ( $\triangleright$ Fig.4). Second, a complete circumferential incision was made. The direction of the incision was determined by the knife type and preference of the endoscopist. Finally, submucosal dissection was performed until the lesion was fully resected. The second lesion was then similarly lifted and resected using the alternate study knife. Intraprocedurally, participants were permitted to reinject the submucosa, remove and clean the knife and endoscope lens, perform washings of the resection field, and ask the assistant to rotate the knife at the participant's discretion. The study coordinator functioned as an assistant/technician to all participants during the procedure, for example with rotation/ control of the knife or repeat submucosal injection. However, in order to allow comparison between participants, no procedural guidance was given during the dissections.

Adverse events were noted by the assistant both during the procedure as well as upon completion of the procedure when the resection area was thoroughly inspected endoscopically. Unintentional incisions were defined as the knife slipping from the working area and causing trauma to another site in the resection area or stomach. Full-thickness perforations were defined as a transmural defect that was externally visible and that resulted in air leakage impairing insufflation. Muscle injury was defined as a breach in the muscle layer without evidence of perforation. Full-thickness perforations were repaired with a through-the-scope clip (and included in the procedure time), whereas muscle injuries were left untreated in the interest of time and resources.

\section{Outcomes}

Outcomes were assessed by the study coordinator during and upon completion of each resection. The primary outcome of efficacy was total procedure time. Procedure time was subdivided into initial mucosal incision (time from mucosal contact with the knife to entry into the submucosal plane), circumferential incision (time from entry into the submucosal plane to completion of the circumferential incision), and submucosal dissection (time from completion of the circumferential incision to complete resection of the lesion), and analyzed separately as part of secondary outcomes. Secondary outcomes also included successful en bloc resection around all border markings, as well as presence of full-thickness perforation and/or muscle injury alone, and unintentional incision.

\section{Survey}

Immediately following ESD of both lesions, participants completed a survey containing six questions assessing the level of difficulty (on a scale of 1 to 5 of increasing difficulty) experienced with use of each knife during the initial incision, remainder of resection (circumferential incision and submucosal dissection), and overall resection.

\section{Statistical analysis}

A sample size was estimated based on the primary outcome of total procedure time, but circumferential and submucosal dissection times were also taken into consideration to ensure ade- 


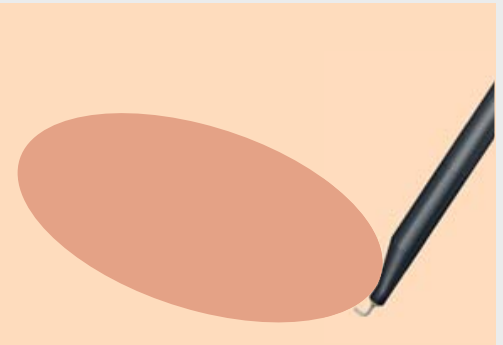

Mucosal incision

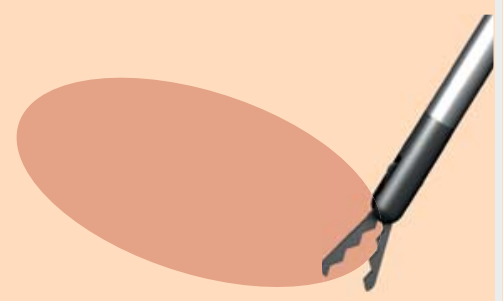

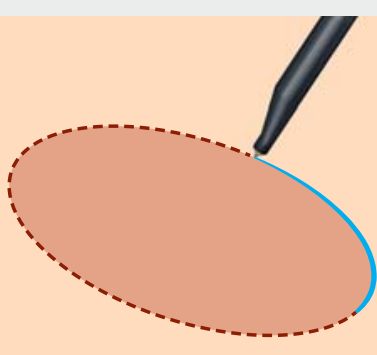

Circumferential incision

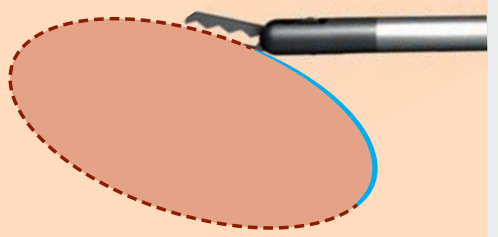

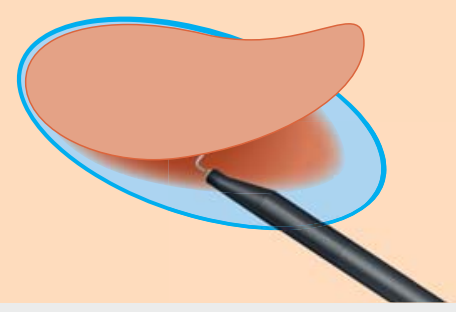

Submucosal dissection

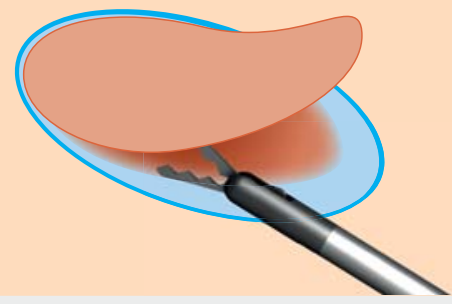

- Fig. 4 Depiction of initial incision, circumferential incision, and submucosal dissection being performed with the conventional knife (top row) and scissor knife (bottom row).

quate statistical power for assessment of secondary outcomes. In a previous study evaluating endoscopists with no ESD experience performing resections of $20 \mathrm{~mm}$ lesions in an ex vivo porcine gastric model, the mean and standard deviation (SD) for total procedure time, circumferential incision time, and submucosal dissection time ranged from 32.6-33.0 (SD 2.20-2.34), 13.3-13.8 (SD 1.22-1.58), and 19.2-19.3 (SD 1.67-2.20) minutes, respectively [11]. We hypothesized that a $20 \%$ reduction using the scissor knife was clinically meaningful. To ensure $95 \%$ power of the paired $t$ test of means for a two-sided significance level of 0.05 , the sample size was calculated as six endoscopists using each knife. We assumed equal SDs between the two knives.

For continuous outcomes, means (SD) were calculated. As this was a crossover study, continuous outcomes were compared using paired $t$ test; categorical outcomes were compared using chi-squared analysis. $P$ values of $<0.05$ were considered statistically significant. All analyses was performed using STATA 14.2 (StataCorp. 2015. Stata Statistical Software: Release 14. College Station, Texas, USA).

\section{Results}

\section{Baseline characteristics}

A total of 10 endoscopists participated in the study: 8 trainees (7 third-year fellows and 1 second-year fellow) and 2 junior general $\mathrm{Gl}$ attending physicians. The median endoscopic experience was 900 procedures (range 430-5000 procedures). All participants had no prior experience of performing ESD and using ESD knives.
Five participants performed the first ESD using the scissor knife and five participants performed the first ESD using the conventional knife. Among a total of 20 ESDs, the majority $(80 \%, 16 / 20)$ were performed in the gastric body (anterior or greater curvature) and in anti-dependent position, with the remainder along the incisura or in the antrum. All ESDs were performed in forward view.

\section{Primary outcome}

\section{Procedure times}

The overall mean total procedure time was 23.5 minutes and without a statistically significant difference between the scissor knife and conventional knife (22.1 [SD 13.3] vs. 24.9 [SD 26.5] minutes; $P=0.65$ ) ( $\triangleright$ Table 1). When compared with the conventional knife, use of the scissor knife was associated with a statistically significant longer time to initial mucosal incision (122.8 [SD 101.0] vs. 33.0 [SD 30.7] seconds; $P=0.02$ ), and although there was a longer time to completion of circumferential incision (13.8 [SD 7.8] vs. 8.4 [SD 11.1] minutes; $P=0.07$ ) this did not reach statistical significance. However, compared with the conventional knife, use of the scissor knife was associated with statistically and clinically significant shorter time to completion of submucosal dissection (6.2 [SD 5.6] vs. 15.6 [SD 15.6] minutes; $P=0.04)$. There was no statistically significant difference in mean total procedure time when the scissor knife was used for the first vs. second resection (20 [SD 11.2] vs. 24.2 [SD 16.1] minutes; $P=0.64$ ); order of use also had no significant effect on subdivided procedure times. 
- Table 1 Outcomes of endoscopic submucosal dissection using the scissor knife and a conventional knife.

\begin{tabular}{|c|c|c|c|}
\hline & Scissor knife ${ }^{1}(n=10)$ & Conventional knife ${ }^{2}(n=10)$ & $P$ value \\
\hline Submucosal injectant volume, mean (SD), mL & $7.8(1.9)$ & $7.5(2.1)$ & 0.71 \\
\hline \multicolumn{4}{|l|}{ Procedure times, mean (SD) } \\
\hline - Total procedure time, minutes & $22.1(13.3)$ & $24.9(26.5)$ & 0.65 \\
\hline - Time to initial incision, seconds & $122.8(101.0)$ & $33.0(30.7)$ & 0.02 \\
\hline - Circumferential incision time, minutes & $13.8(7.8)$ & $8.4(11.1)$ & 0.07 \\
\hline - Submucosal dissection time, minutes & $6.2(5.6)$ & $15.6(15.6)$ & 0.04 \\
\hline En bloc resection, \% (n/N) & $90.0(9 / 10)$ & $70.0(7 / 10)$ & 0.26 \\
\hline Successful resection around all border markings, \% $(n / N)$ & $90.0(9 / 10)$ & $90.0(9 / 10)$ & $>0.99$ \\
\hline \multicolumn{4}{|l|}{ Adverse events, \% (n/N) } \\
\hline - Perforation or muscle injury & $10.0(1 / 10)$ & $70.0(7 / 10)$ & $<0.01$ \\
\hline - Perforation & $10.0(1 / 10)$ & $30.0(3 / 10)$ & 0.26 \\
\hline " Muscle injury & $10.0(1 / 10)$ & $60.0(6 / 10)$ & 0.02 \\
\hline - Unintentional incision & $10.0(1 / 10)$ & $70.0(7 / 10)$ & $<0.01$ \\
\hline - No. of unintentional incision/resection, median (IQR) & $0(0-0)$ & $2(0-3))$ & 0.06 \\
\hline $\begin{array}{l}\text { SD, standard deviation; IQR, interquartile; range. } \\
{ }^{1} \text { Clutch Cutter; Fujifilm Co., Tokyo, Japan. } \\
{ }^{2} \text { HookKnife; Olympus Endotherapy, Tokyo, Japan. }\end{array}$ & & & \\
\hline
\end{tabular}

\section{Secondary outcomes}

Efficacy

Both the scissor knife and the conventional knife were associated with $90 \%$ successful resection around all border markings ( $\triangleright$ Table 1). A higher proportion of en bloc resection was achieved with the scissor knife compared with the conventional knife without reaching statistical significance ( $90 \%$ vs. $70 \% ; P=$ $0.26)$. Despite not reaching a statistical significance in en bloc resection, a $20 \%$ difference is potentially clinically meaningful. Evidently, in two cases using the conventional knife, inadvertent disruption to the mucosal side of the lesion occurred during submucosal dissection, which was not observed with use of the scissor knife.

\section{Adverse events}

During ESD with the conventional knife, one or more perforations (range $1-3$ ) occurred in 3 cases $(30 \%, 3 / 10)$, and muscle injuries (range $1-4)$ occurred in 6 cases $(60 \%, 6 / 10)$ ( Table 1 ). During ESD with the scissor knife, one perforation occurred in 1 case $(10 \%, 1 / 10)$, and all muscle injuries $(n=4)$ occurred in the same case $(10 \%, 1 / 10)$. Notably, the participant performing ESD in this case had the second lowest volume of endoscopy experience of the study group at the time of participation (total endoscopic procedures 572). All perforations and muscle injuries in both groups occurred during the submucosal dissection phase of resection. Compared with the conventional knife, use of the scissor knife was associated with a statistically significant and likely clinically meaningful lower proportion of perforation and/or muscle injury (10\% vs. $70 \% ; P<0.01)$ and muscle injury alone $(10.0 \%$ vs. $60.0 \% ; P=0.02)$, but not perforation alone ( $10 \%$ vs. $30 \%$; $P=0.26$ ). Use of the scissor knife was also associated with a statistically significantly lower proportion of unintentional incision (10\% vs. $70.0 \% ; P<0.01)$ ( $>$ Table 1 ).

\section{Survey}

There was no statistically significant difference in perception of difficulty level using the scissor knife vs. conventional knife for the overall resection (mean score 3.2 [SD 1.1] vs. 3.3 [SD 1.6]; $P$ $=0.85$ ), the initial mucosal incision (mean score 3.2 [SD 1.5] vs. 2.1 [SD 1.1]; $P=0.10$ ), or completion of the circumferential incision/submucosal dissection (mean score 2.4 [SD 1.0] vs. 3.6 [SD 1.3]; $P=0.07)$.

\section{Discussion}

ESD is a valuable technique to resect large superficial GI lesions but remains technically challenging. The steep learning curve and safety profile of ESD are an impediment to trainees and limit its widespread adoptability [1]. Recent efforts to enhance the performance and safety of ESD have included various ESD accessories that improve tissue visualization and modifications in knife design including a scissor-type category of knives. However, experience with these knives in novices, particularly in Western regions, is lacking. In this ex vivo study comparing the use of the Clutch Cutter scissor knife with a conventional HookKnife ESD knife among novices without experience in ESD, we made several notable observations. Use of the scissor knife was associated with a lower proportion of significant adverse events (i.e. perforation and/or muscle injury). In addition, the 
scissor knife did not prolong the overall procedure time and, in particular, reduced the submucosal dissection time. These findings may have important implications for the trainee performing ESD.

Perforation remains one of the most feared complications of ESD, particularly for the trainee. Although we did not find a significant difference in the rates of perforation between the two knife groups, the rate of muscle injury alone was significantly reduced in the scissor knife group ( $\mathbf{F i g . 5 )}$ ). Muscle injury carries similar clinical implications to those of perforation as it increases the risk of delayed perforation, requires antibiotics and endoscopic closure (lengthening the procedure time), may increase the complexity of the remainder of the resection, and may impact the decision to hospitalize the patient for observation.

All perforations and muscle injuries in the current study occurred during submucosal dissection. This is not surprising considering submucosal dissection is often the most technically challenging aspect of ESD for trainees and experts alike. This is primarily due to the difficulty in maintaining adequate exposure of the submucosa to facilitate safe dissection along the submucosal plane. Indeed, there are several new promising accessories and techniques that aim to assist in tissue retraction [12-14]. The scissor knife may offer alternative and adjunctive means of reducing significant adverse events during submucosal dissection. Scissor-type knives grasp the tissue prior to application of the current, providing the endoscopist with an opportunity to verify the position and technique and, if necessary, make adjustments before committing to cutting. Grasping also allows manipulation of the tissue, which may improve the visual field but also allows the tissue to be lifted away from the muscle layer to prevent inadvertent deep injury. Notably, the outer edges of the scissors including the blunt tip are insulated, providing additional protection in this regard. We also observed that once the scissor knife was initially oriented for submucosal dissection, it infrequently required adjustment and the axis of the scissor likely helped the novice to maintain constant orientation along the submucosal plane.

The only case during which perforation or muscle injury occurred with the scissor knife was performed by a participant who had the second lowest volume of endoscopy experience. The same participant also demonstrated multiple perforations and muscle injuries using the conventional knife, which was randomized for first use. These adverse events with use of both knives may therefore be related to deficiencies in the participant's basic endoscopic skill set and/or understanding of ESD rather than the characteristics of a particular knife. This underscores the importance of a foundation that includes endoscopic (including EMR) experience and understanding of ESD theory.

Use of the scissor knife significantly improved submucosal dissection time, but this appeared to be offset by a trend toward longer initial incision and circumferential incision times; hence, overall procedure time was not significantly prolonged. Improved submucosal exposure and orientation, as noted above, likely contribute to more efficient dissection as well. Grasping of tissue with forceps also appeared to provide great-

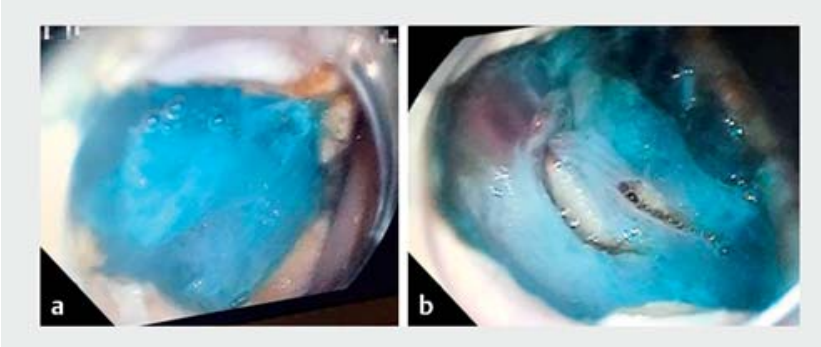

- Fig. 5 Examples of resection beds following endoscopic submucosal dissections with the scissor knife (a) and the conventional knife (b) by the same participant. There is no evidence of muscle injury or perforation with the scissor knife, whereas two muscle injuries are apparent with the conventional knife.

er stability and maneuverability and was more intuitive in the hands of the novice compared with the conventional knife. Scissor-type knives are mechanistically similar to biopsy forceps, which is among the first endoscopic accessories used by trainees and most commonly employed by endoscopists. The conventional knife requires use of a relatively unfamiliar technique for general endoscopists. Although there was a trend in participant preference for the scissor knife following the initial incision, this may have been confounded by consolidating feedback for circumferential incision and submucosal dissection given that the scissor knife was less efficient for the former and more efficient for the latter.

The significantly longer initial incision time with the scissor knife appeared to be related to difficulty in grasping a sufficient fold (i.e. two layers) of mucosa. This is despite the use of the longer knife $(5.0 \mathrm{~mm})$ instead of the shorter length $(3.5 \mathrm{~mm})$. Repeated grasping and cutting cycles were often necessary to create an initial incision sufficient to expose underlying submucosa. It is possible that the ex vivo porcine model, in which the gastric mucosa is thickened, may have exacerbated this. Using the scissor knife initially in an open position pressed against the mucosa to facilitate an initial mucosal incisional hole may be helpful. Subsequent circumferential incision was less redundant from this standpoint as incision of only a single layer of mucosa was necessary. However, novices in the current study encountered difficulty in repeatedly realigning the axis of the scissors with the desired mucosal cutting direction, particularly toward the distal aspect of the lesion; this appeared less cumbersome with the conventional knife. In addition, unlike the scissor knife, the conventional knife did not require as much support from the assistant in rotating and opening/closing the knife. Alternate electrosurgical settings (such as Endocut I) may have also allowed smoother cutting in this setting with the scissor knife.

These findings have important implications for both trainees and supervisors of ESD. The Clutch Cutter and scissor category of knives may offer a more intuitive approach to aspects of ESD than the HookKnife and other similarly designed knives. Given its capability for marking, initial mucosal incision, dissection, and hemostasis, the Clutch Cutter scissor knife may also reduce 
the number of accessories trainees must initially learn to perform ESD. Furthermore, grasping tissue prior to applying the electrosurgical current allows stepwise approval by the supervising endoscopist and adjustment, if necessary, without harm. This may be particularly beneficial in guiding the trainee through more challenging portions of dissection. Indeed, recent studies from Japan comparing the Clutch Cutter to IT2 knife (Olympus) and needle-knife with waterjet function among nonexperts demonstrated that Clutch Cutter use was associated with higher self-completion rates for ESD in the stomach and colon $[8,15]$.

Due to the ex vivo study design, it is unclear whether the results in this study apply to the in vivo setting where additional procedural variables coexist such as hemostasis, respiratory motion, peristalsis, and need for reinjection of the submucosa. Hemostasis in particular can pose a major barrier to completing an ESD procedure, although in a retrospective study, higher self-completion of gastric ESD with the Clutch Cutter was attributed to the knife's hemostatic efficacy. Review of published videos and our own experience with this knife in vivo have found that hemostasis is actually much improved as the device allows coaptive coagulation of vessels very similar to the Coagrasper (Olympus America Inc., Center Valley, Pennsylvania, USA). However, further study comparing scissor-type ESD knives with other types in an in vivo setting is warranted.

This study has several other limitations worth highlighting. Our findings apply to novices and it is unclear whether our findings are generalizable to trainees further along in the learning curve. Similarly, we studied the most basic ESD lesions $(2 \mathrm{~cm}$ in diameter, gastric body, forward-view resection), and findings may differ for larger resections in other locations of the stomach or GI tract. We also cannot be certain that these results are generalizable to other scissor-type ESD knives (i.e. Stag Beetle knife; Sumitomo Bakelite Co., Tokyo, Japan) but would assume so based on similarities in design. Similarly, the HookKnife, which was chosen because of its rotational and traction properties, may carry increased risk in inexperienced hands and may not be generalizable to straight knives such as the DualKnife (Olympus) or FlushKnife (Fujifilm). It should also be noted that the electrosurgical settings selected for this ex vivo animal study are not applicable to the in vivo (including human) setting. Finally, given the crossover design of the study, there might be a carryover effect, as the endoscopist's skill could improve with the second ESD; however, there was no significant difference in the procedure time when the Clutch Cutter was used for the first or second resection.

Among novices performing ESD on an ex vivo animal model, use of the Clutch Cutter scissor knife was associated with a significantly lower proportion of adverse events without prolonging the procedure time. The Clutch Cutter may improve the safety of ESD, at least initially among trainees. Further investigation is needed to better define its role in the trainee's path to learning ESD.

\section{Competing interests}

Kenneth K. Wang, Research funding from Fujinon Medical

Funding

Van Cleve Endowed Professorship NCI U01 CA182940

\section{References}

[1] Schlachterman A, Yang D, Goddard A et al. Perspectives on endoscopic submucosal dissection training in the United States: a survey analysis. Endosc Int Open 2018; 06: E399-E409

[2] Kotzev Al, Yang D, Draganov PV. How to master endoscopic submucosal dissection in the USA. Dig Endosc 2019; 31: 94-100

[3] Okumoto T, Kuwai T, Yamaguchi T et al. OP240: Efficacy, safety and clinical outcomes of endoscopic submucosal dissection using a scissors-type knife for early esophageal neoplasms. United Eur Gastroenterol J 2015; 3: (Suppl. 05): A78

[4] Akahoshi K. A new breakthrough: ESD using a newly developed grasping type scissor forceps for early gastrointestinal tract neoplasms. World J Gastrointest Endosc 2010; 2: 90

[5] Akahoshi K, Akahane H, Motomura Y et al. A new approach: endoscopic submucosal dissection using the Clutch Cutter ${ }^{\circledR}$ for early stage digestive tract tumors. Digestion 2012; 85: 80-84

[6] Akahoshi K, Honda K, Motomura Y et al. Endoscopic submucosal dissection using a grasping-type scissors forceps for early gastric cancers and adenomas. Dig Endosc 2011; 23: 24-29

[7] Yoshida N, Dohi O, Inoue K et al. Efficacy of scissor-type knives for endoscopic mucosal dissection of superficial gastrointestinal neoplasms. Dig Endosc 2020; 32: 4-15

[8] Dohi O, Yoshida N, Terasaki K et al. Efficacy of Clutch Cutter for standardizing endoscopic submucosal dissection for early gastric cancer: a propensity score-matched analysis. Digestion 2019; 100: 201-209

[9] Hayashi Y, Esaki M, Suzuki S et al. Clutch Cutter knife efficacy in endoscopic submucosal dissection for early gastric neoplasms. World J Gastrointest Oncol 2018; 10: 487-495

[10] Kuwai T, Yamaguchi T, Imagawa H et al. Endoscopic submucosal dissection for early esophageal neoplasms using the stag beetle knife. World J Gastroenterol 2018; 24: 1632-1640

[11] Kato M, Gromski M, Jung Y et al. The learning curve for endoscopic submucosal dissection in an established experimental setting. Surg Endosc 2013; 27: 154-161

[12] Ge PS, Thompson CC, Jirapinyo P et al. Suture pulley countertraction method reduces procedure time and technical demand of endoscopic submucosal dissection among novice endoscopists learning endoscopic submucosal dissection: a prospective randomized ex vivo study. Gastrointest Endosc 2019; 89: 177-184

[13] Hashimoto R, Hirasawa D, Iwaki T et al. Usefulness of the S-O clip for gastric endoscopic submucosal dissection (with video). Surg Endosc 2018; 32: 908-914

[14] Yoshida M, Takizawa K, Igarashi K et al. OP130: Efficacy of endoscopic submucosal dissection with dental floss clip traction for gastric epithelial neoplasia: a pilot study. United Eur Gastroenterol J 2015; 3: (Suppl. 05): A43

[15] Yamashina T, Takeuchi Y, Nagai K et al. Scissor-type knife significantly improves self-completion rate of colorectal endoscopic submucosal dissection: single-center prospective randomized trial. Dig Endosc 2017; 29: 322-329 